\title{
Les couronnes piliers hybrides transvissées sur implant : protocole rigoureux de collage des embases titane
}

\section{Hybrid abutment crowns transmitted on implant: rigorous protocol for bonding titonium bases}

\section{Résumé}

\section{MOTS-CLEFS :}

- Couronne pilier hybride, couronne implantaire transvissée, disilicate de lithium, pilier implantaire, CFAO

\section{KEYWORDS:}

- Hybrid abutment crown, screw retained implant crown, lithium disilicate, implant abutment, CFAO
Les couronnes piliers hybrides appelées également couronnes transvissées et les piliers hybrides unitaires sur implants sont réalisables par CFAO au cabinet dentaire. Après l'empreinte optique et la conception sur ordinateur, la prothèse est usinée dans un bloc de disilicate de lithium percé, adapté aux dimensions d'une embase en titane préfabriquée (TiBase ${ }^{\circledR}$ ) qui assure la connexion avec l'implant. Le collage de l'embase dans la couronne et la finition de la prothèse doivent être réalisées soigneusement pour assurer sa biocompatibilité. Les contrôles cliniques et radiographiques des prothèses placées depuis les quatre ans d'utilisation du procédé attestent de sa fiabilité.

\author{
Abstract
}

Hybrid abutments crowns, usually called screw retained crowns and single hybrid abutments can be made by CFAO in dental office. After optical impression and computerized design, prosthesis is milled in a perforated lithium disilicate block, adapted to a prefabricated titanium base (TiBase ${ }^{\circledR}$ ) who will be connected to implant. Bonding and finishing procedures of the prosthesis must be thoroughly achieved to obtain biocompatibility. Workflow reliability is attested by clinic and radiographic controls of prosthesis installed during four last years.

$\operatorname{AOS} n^{\circ} 283-2017$

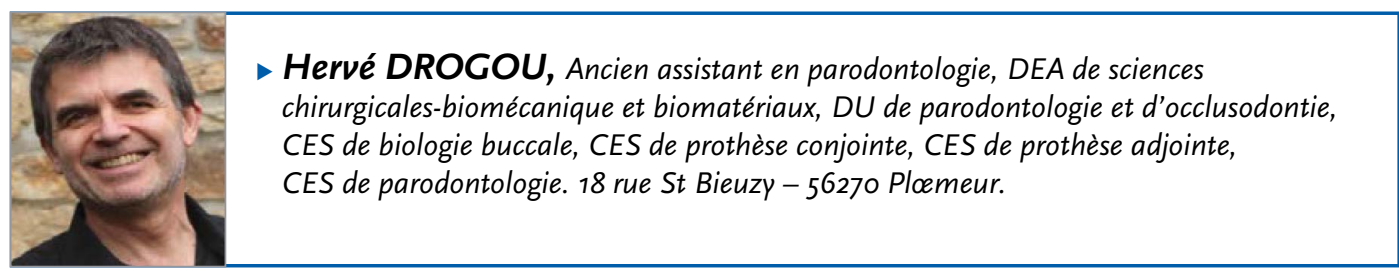

Les techniques de CFAO au fauteuil sont utilisables pour fabriquer des prothèses implanto-portées unitaires. Elles offrent désormais la possibilité d'usiner des couronnes monolithiques percées et des piliers en céramique puis de les assembler par collage sur une embase en titane (1). Ces éléments prothétiques sont appelés couronnes piliers hybrides pour les couronnes transvissées (cas cliniques $n^{\circ} 1,2$ et 3 ) et piliers hybrides pour les infrastructures destinées aux prothèses scellées (cas clinique $n^{\circ} 4$ et 5 ).

Cette technique, déjà utilisée de manière artisanale à partir de l'empreinte (optique ou traditionnelle) d'un pilier du commerce, a été récemment optimisée grâce à la préparation d'éléments calibrés par usinage industriel pour en accentuer la précision. (Fig. 1) Parallèlement, l'empreinte optique intrabuccale 
directe du corps implantaire a été rendue possible par l'utilisation d'un transfert d'empreinte (scan post) associé à un repère optique (scan body). (Cas clinique $\mathrm{n}^{\circ} 1,4$ et 5 ). La commercialisation de ce système a débuté en Europe au printemps 2013 après plusieurs années de recherche sur le matériel et sur le logiciel Cerec $^{\circledR}$. Les USA ont délivré leur agrément FDA en octobre 2013 (2).

Bien qu'il n'y ait pas encore d'étude clinique sur le long terme, la technique connaît un fort engouement chez les praticiens et prothésistes équipés en CFAO. Signe des temps, la fiabilité du système sur les quatre ans d'utilisation a été approuvée par les praticiens sur les réseaux sociaux avant la publication de statistiques dans la littérature scientifique.

La qualité esthétique de la prothèse est assurée par les propriétés optiques du disilicate de lithium qui peut ensuite soit être recouvert de céramique de maquillage soit être aménagé par soustraction pour laisser la place à une stratification de céramique feldspathique selon la technique dite du cut-back. (Cas clinique $\mathrm{n}^{\circ} 3$ )

La qualité et le comportement des tissus périimplantaires dépendent de la qualité des éléments prothétiques : parallèlement à l'essor des couronnes céramiques transvissées et des piliers céramiques sur implants, la facilité à personnaliser l'émergence sous gingivale des prothèses par CFAO contribue à leur intégration biologique et esthétique. (Cas clinique $n^{\circ} 1$ ) (3) L'assemblage d'un pilier ou d'une couronne pilier hybride associe trois éléments : l'élément en céramique (obtenu par usinage d'un bloc céramique), la tibase (embase en titane transvissée) et le système de collage. \Le bloc en céramique prêt à être usiné dans une usineuse (Fig. 1 et 2 ) est obtenu industriellement à partir d'un lingotin aplani sur une face et percé en son centre aux dimensions de la base titane. Les matériaux disponibles sont : l'IPS e.max CAD ${ }^{\circledR}$ distribué par la société Ivoclar Vivadent ${ }^{\oplus}$ qui a développé le procédé, l'Enamic ${ }^{\circledR}$, céramique hybride distribuée par la société $\mathrm{Vita}^{\circledR}$ et l'oxyde de zirconium InCoris ${ }^{\oplus}$ de Dentsply-Sirona ${ }^{\circledR}$. Il existe également des blocs en PMMA (Polyméthacrylate de méthyle), destinés aux prothèses temporaires : le Telio $\mathrm{CAD}^{\circledR}$ d'Ivoclar Vivadent ${ }^{\oplus}$.

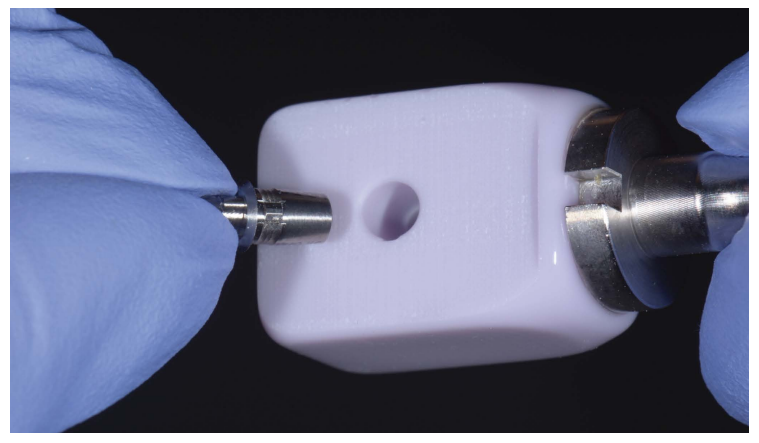

$\triangle$ Fig. 1 : TiBase $^{\circ}$ et bloc IPS e.max $\mathrm{CAD}^{\circledR}$ avant usinage.
Les tests de résistance in vitro de piliers hybride en zircone ou en disilicate de lithium n'ont pas démontré de différence entre les deux matériaux. Après la mise sous contraintes des éprouvettes, des déformations plastiques interviennent sur le métal, sans fracture de la céramique. Les piliers hybrides se sont avérés plus résistants que les piliers en zircone seule (4).

I L'embase en titane comporte une extrémité avec un détrompeur adapté à l'orifice du bloc céramique (lui aussi doté d'un détrompeur) et l'autre extrémité adaptée à la connexion implantaire (Fig. 1 et 2). Même s'il existe une variété d'embase pour se connecter aux implants les plus répandus, beaucoup de plateformes implantaires n'ont pas encore d'embases en titane disponibles pour cette technique. Fabriquées à l'origine par la société SIRONA ${ }^{\oplus}$ sous l'appellation TiBase ${ }^{\varpi}$, ces éléments adaptés aux blocs à usiner sont maintenant proposés par plusieurs fabricants.

La colle recommandée et mise au point par le fabricant de céramique est fluide et opaque pour assurer un joint le plus fin possible et masquer le titane gris lorsque l'épaisseur de céramique est faible. Le Multilink Hybrid Abutment ${ }^{\circledast}$ a été développé par Ivoclar Vivadent ${ }^{\circledR}$ pour cet usage. L'étude de Weyhrauch et coll. (5) compare plusieurs produits de collage sans trouver de différences significatives et elle confirme la solidité des prothèses en céramique monolithique collées sur base titane. Tous les tests de fatigue ont abouti à la fracture des vis ou des implants, sans endommager la prothèse.

Notons cependant que les TiBases ${ }^{\circledast} \mathrm{S}$ ne sont disponibles que pour quelques références d'implants. Cela contre-indique la technique pour des incisives mandibulaires ou latérales maxillaires étroites.

La réponse tissulaire à cet assemblage dépend non seulement de la biocompatibilité respective de ces trois matériaux, de leurs états de surface $(6,7,8)$,

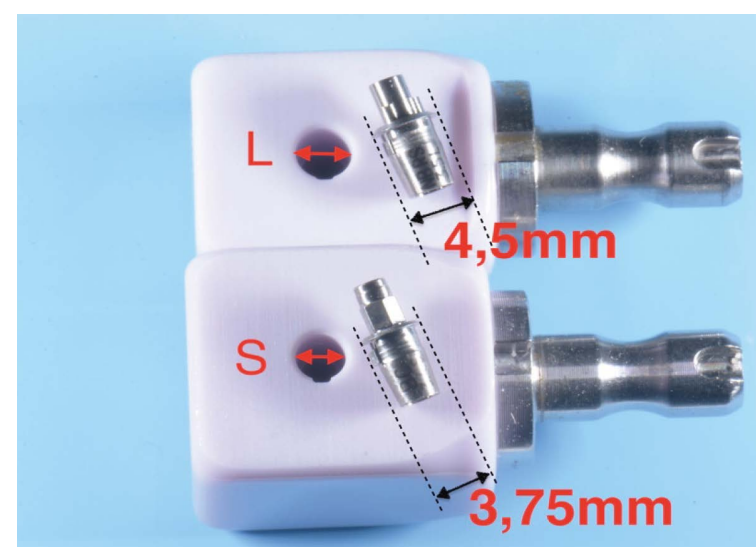

$\triangle$ Fig. 2 : les blocs IPS e.max $\mathrm{CAD}^{\circledR}$ sont disponibles avec deux diamètres d'orifices différents, correspondant à deux modèles de TiBases $^{\oplus}$ : $\mathrm{S}$ ou L. Cette dimension conditionne la largeur cervicale de la prothèse. Les TiBases ${ }^{\circledR} \mathrm{S}$ permettent de réaliser des prothèses de $3,75 \mathrm{~mm}$ de diamètre cervical minimum, contre $4,5 \mathrm{~mm}$ minimum pour les $\mathrm{TiBases}^{\circledast} \mathrm{L}$. 
mais également de la finesse du joint de colle et de la propreté de la prothèse dans son émergence sous gingivale.

Les études sur la biocompatibilité du titane ne manquent pas, par contre, la plupart des études concernant la solidité et la biocompatibilité du disilicate de lithium testent le matériau sous sa forme pressée au laboratoire de prothèse et non sous la forme destinée à la CFAO pressée préalablement en usine. Des études complémentaires sont nécessaires.

Avant collage, la précision de ces éléments usinés est très satisfaisante et la coaptation est parfaite au contrôle visuel. Cependant, il importe de respecter des étapes essentielles de manipulation dans le procédé de fabrication. Le collage et le polissage sont opérateur dépendants. Ils sont réalisés au cabinet dentaire ou au laboratoire de prothèse selon l'organisation du praticien. L'examen visuel avec aides optiques doit confirmer l'obtention d'un joint de colle quasiment invisible.

Le protocole détaillé est précisé dans la documentation du fabricant. Quelques étapes indispensables au succès sont illustrées ici (Fig. 3 à 10).

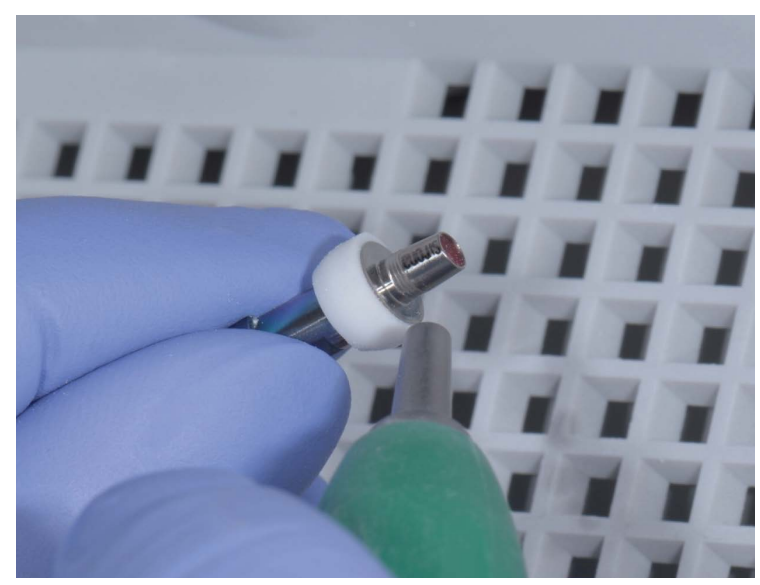

$\triangle$ Fig. 3 : la TiBase ${ }^{\varpi}$ est vissée sur une réplique d'implant et le collet est protégé par une bague en silicone d'identification d'instruments. Le sablage à l'oxyde d'aluminium à 50 microns est effectué dans une enceinte à aspiration pour éviter l'inhalation de la poudre par l'opérateur.

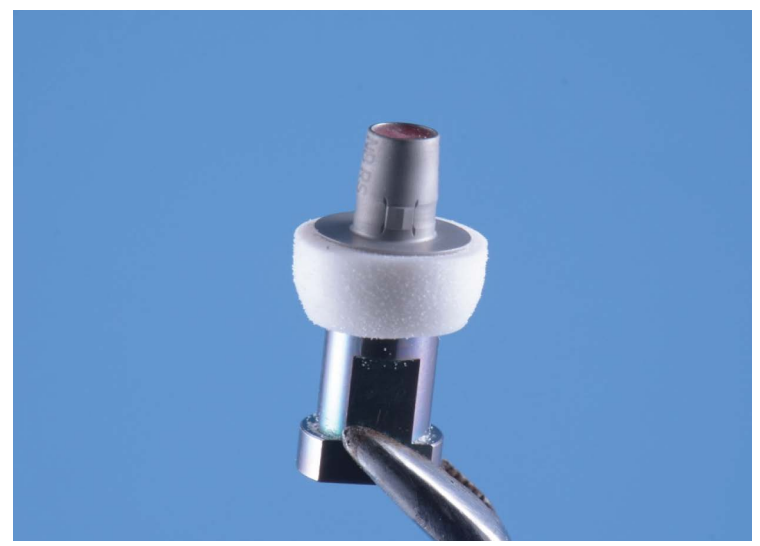

$\triangle$ Fig. 4 : la TiBase ${ }^{ø}$ est convenablement dépolie. Le puits d'accès à la vis a été préalablement obturé à la cire à boxing.
Liste des étapes du collage :

$\checkmark$ essayage de la TiBase ${ }^{\circledR}$ dans la céramique cristallisée I vissage de la TiBase ${ }^{\bowtie}$ sur une réplique d'implant $\checkmark$ insertion d'une bague de silicone de protection $\checkmark$ sablage à l'oxyde d'aluminium de la TiBase ${ }^{\circledR}$ en protégeant le col sous gingival

nettoyage de la céramique par un jet de vapeur $\checkmark$ obturation du puits de vis par de la cire tendre $\checkmark$ mordançage de la céramique à l'acide fluorhydrique 20 ' et rinçage

I application de silane sur le titane

$\checkmark$ mélange et application de la colle sur le titane $\checkmark$ insertion de la TiBase ${ }^{\bowtie}$ dans la céramique en orientant le détrompeur dans l'encoche

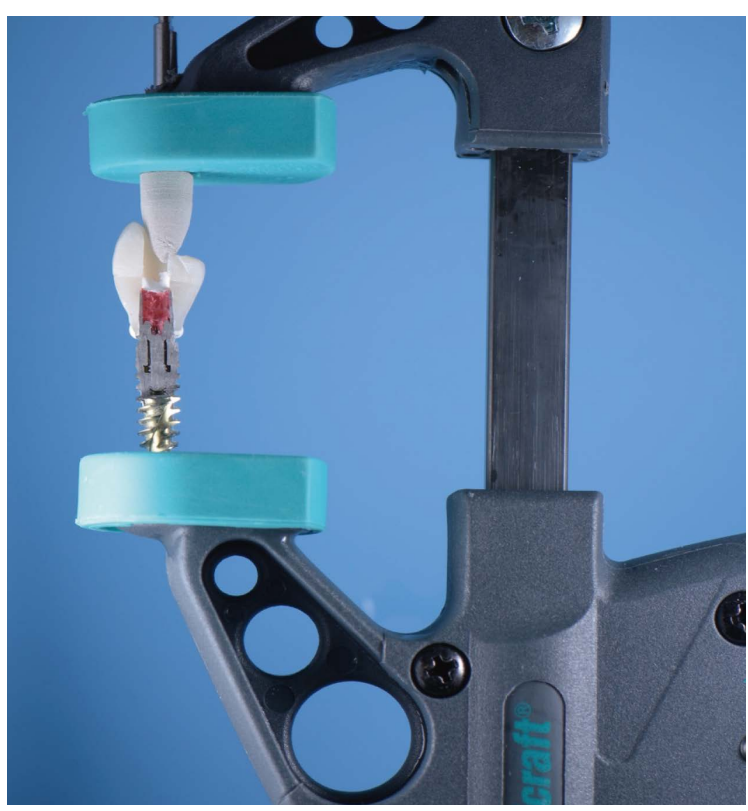

$\triangle$ Fig. 5 : coupe longitudinale de l'assemblage. Après traitement du puits de céramique à l'acide fluorhydrique et silanisation, la TiBase ${ }^{\star}$ et la couronne sont collées avec le Multilink Hybrid Abutment ${ }^{\oplus}$. L'ensemble est pressé fermement pendant 7 minutes dans un petit serre-joint modifié. Pour éviter une pression décentrée par la longueur d'une cuspide, le serre-joint est modifié par l'adjonction d'une pointe monté de polissage. La pression est ainsi exercée dans l'axe longitudinal au niveau de l'orifice de vissage.

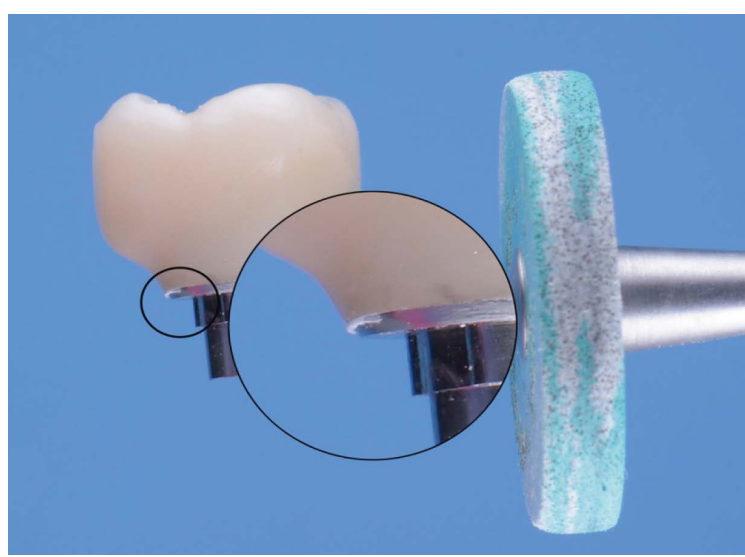

$\triangle$ Fig. 6 : les bords de céramique et le joint de collage sont polis à l'aide de disques en silicone de granulométrie décroissante pour obtenir un poli miroir. 
pression digitale forte

nettoyage des excès à la micro brush

$\checkmark$ pression à l'aide d'un serre joint modifié pour exercer une pression verticale dans l'axe de l'implant

$\checkmark$ remontée de la bague silicone pour lisser le joint et le placer à l'abri de l'oxygène

I attente de polymérisation 7 minutes

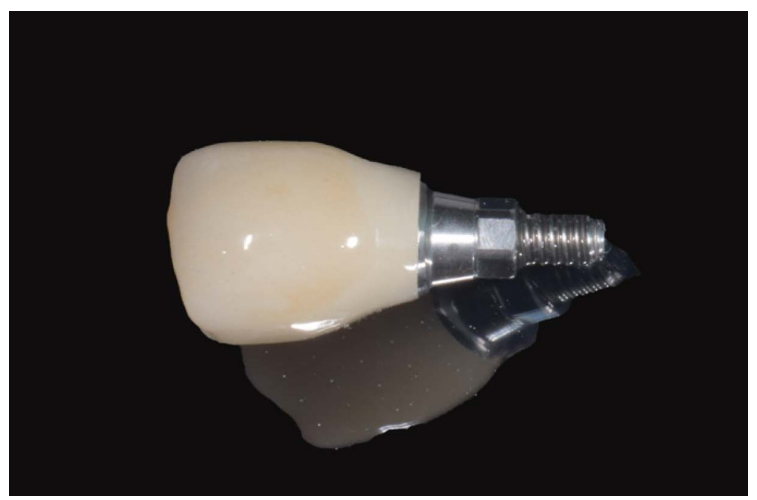

$\triangle$ Fig. 7 : le joint céramique/titane doit être réduit au minimum pour éviter une rétention de bactéries. La céramique et le titane sont polis soigneusement après le collage. La connectique est ensuite débarrassée de toute trace de colle par un balayage à l'aéro-polisseur au carbonate de calcium de faible granulométrie (poudre Air-N-Go Acteon $^{\circ}$ ) puis désinfectée à la vapeur sous pression.

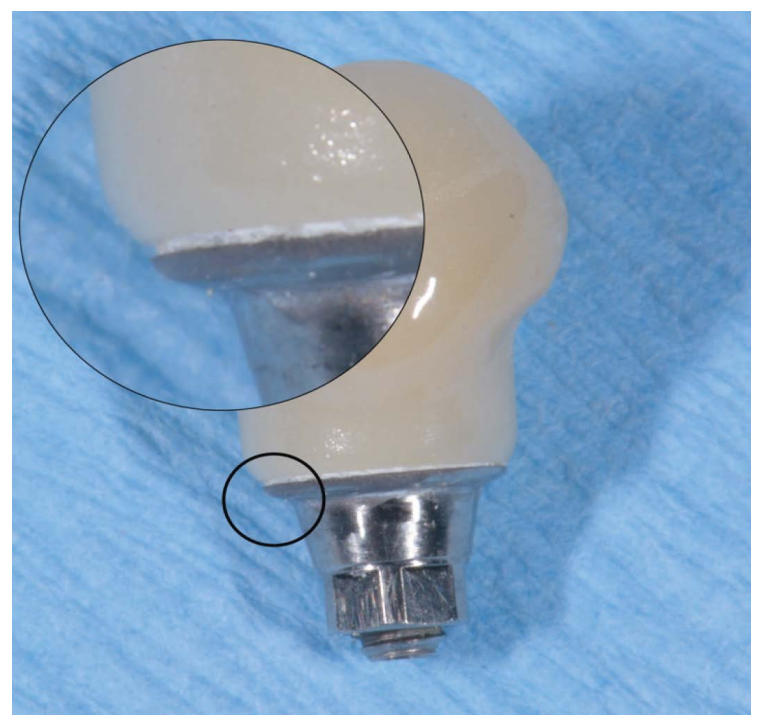

$\triangle$ Fig. 9 : Exemple d'assemblage défectueux. Plusieurs erreurs de fabrication se cumulent dans la portion sous gingivale de cette couronne pilier hybride qui contre indiquent son placement en prothèse d'usage. Le joint de collage est très épais et la céramique est à distance de la TiBase ${ }^{\circ}$. L'état de surface rugueux est favorable à l'adhésion bactérienne. Le glaçage de la céramique nappe la portion sous gingivale.

\section{CAS CLINIQUES}

Les constatations cliniques sur les exemples proposés ici sont en faveur d'une bonne tolérance de ces prothèses. L'absence d'inflammation gingivale, la stabilité
I adoucissement et polissage des limites

$\checkmark$ nettoyage à l'aéro polisseur à la poudre de bicarbonate très fine

nettoyage final à la vapeur

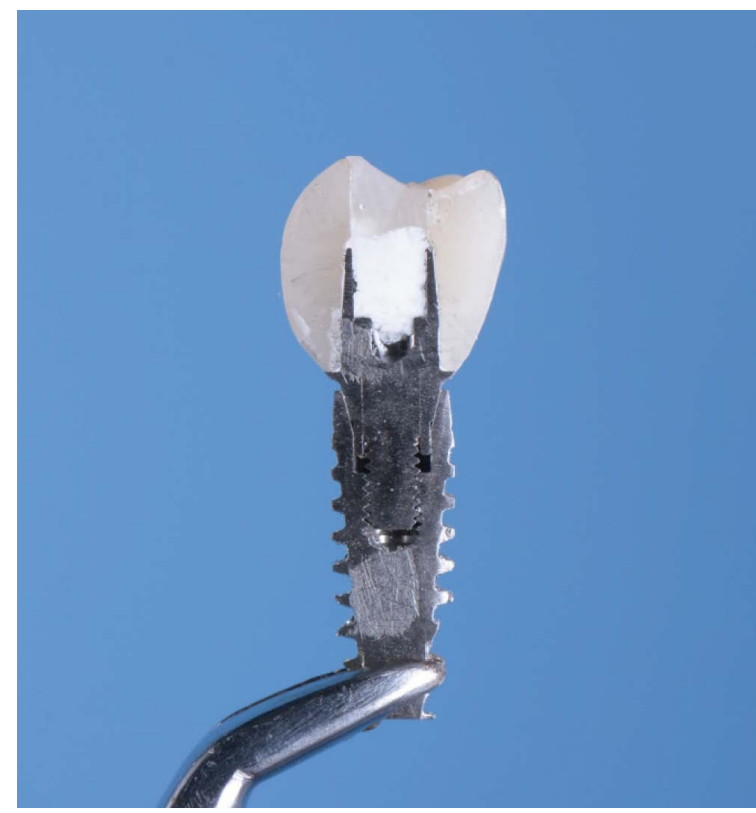

$\triangle$ Fig. 8: section longitudinale d'une couronne pilier hybride sur implant factice NobelActive RP. Après essayages et ajustages éventuels, la prothèse est insérée dans l'implant puis vissée au torque de $35 \mathrm{nw} / \mathrm{cm}$. Le puits de vissage est obturé par un ruban de polytétrafluoroéthylène tassé (9) recouvert d'un composite postérieur à faible taux de rétraction collé après conditionnement de la céramique à l'aide de Total Etch $^{\oplus}$ de Ivoclar Vivadent ${ }^{\circ}$

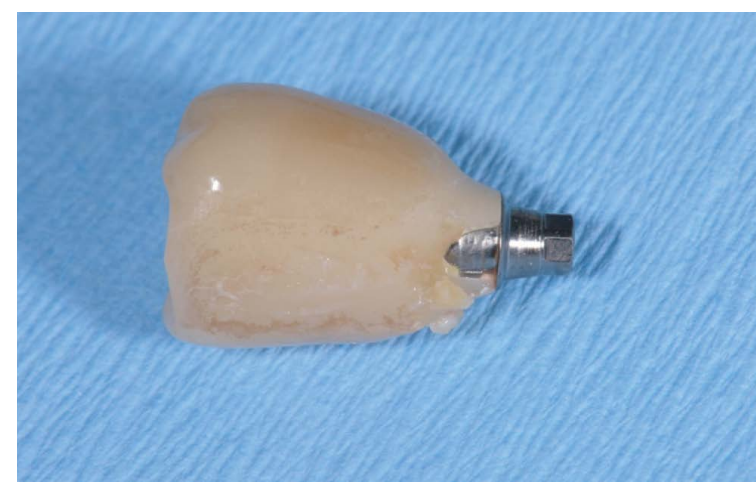

$\triangle$ Fig. 10 : Échec survenu après 3 mois sur un pilier couronne hybride en raison d'une erreur de protocole. Le titane n'a pas été sablé et la quantité de colle est insuffisante. Il s'agit d'un des deux seuls échecs connus survenus en 3,5 ans sur 524 réalisations au cabinet dentaire. L'autre échec est survenu sur un pilier de bridge pour la même raison.

du niveau osseux péri-implantaire sont les critères dont disposent les praticiens pour juger de la bonne tolérance de leurs prothèses. 
Cas clinique $n^{\circ} 1$ (Fig. 11a, b, c, d)

La première couronne pilier hybride placée par notre équipe en septembre 2013. Le contrôle clinique et radiographique atteste d'une bonne tolérance de la prothèse.

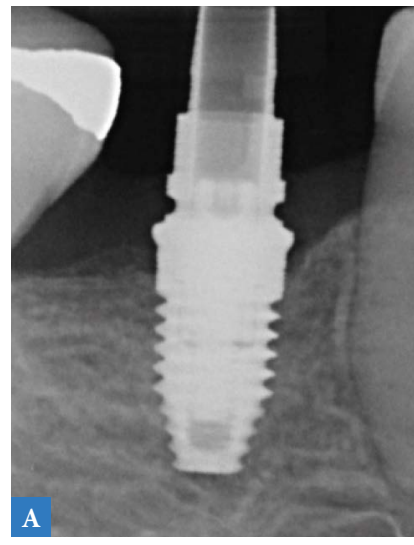

$\triangle$ Fig. 11a : radiographie du transfert d'empreinte (scan post) vissé sur l'implant.

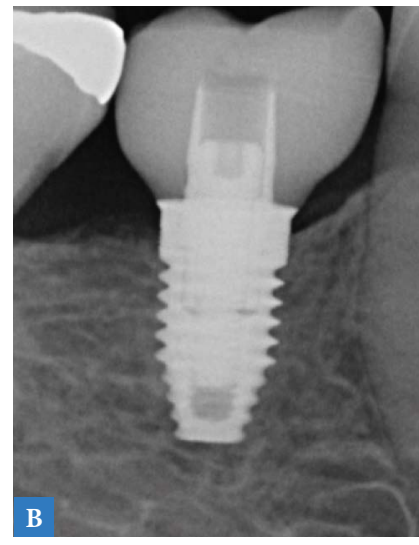

$\triangle$ Fig. 11b : pose de la prothèse en septembre 2013

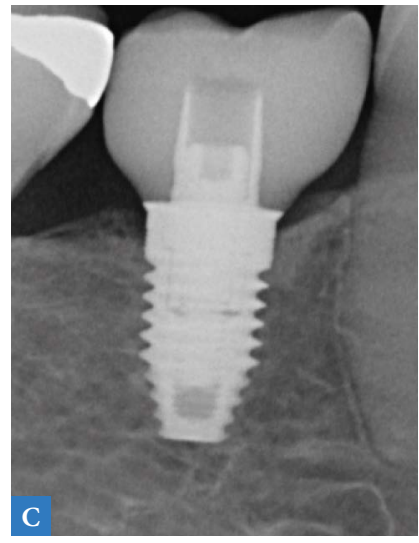

$\triangle$ Fig. 11c : contrôle radiographique en février 2017

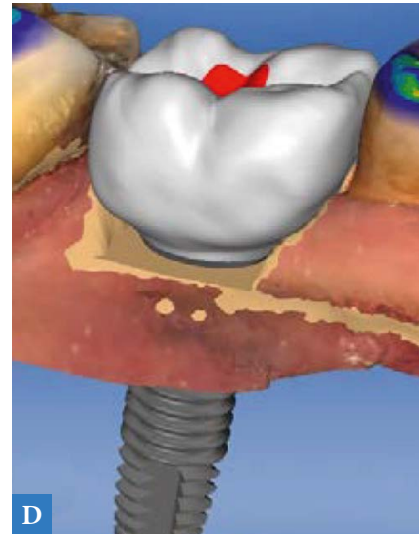

$\triangle$ Fig 11d : conception de la prothèse avec le logiciel Cerec $4.3^{\circ}$.

\section{Cas clinique $n^{\circ} 2$ (Fig. 12a, b, c et Fig. 13)}

Couronne pilier hybride placée en février 2014.

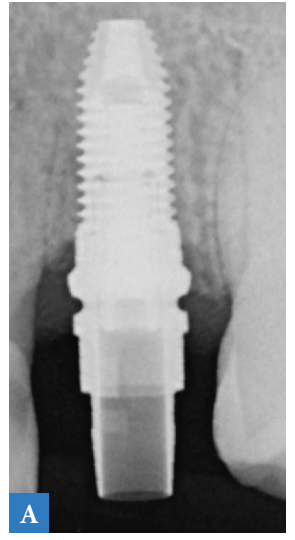

$\triangle$ Fig. 12a : radiographie du transfert d'empreinte (scan post) vissé sur l'implant.

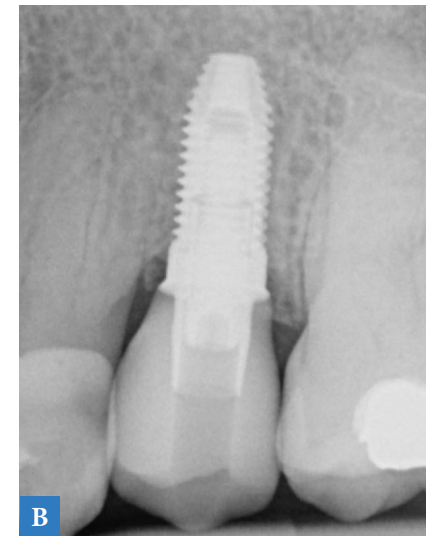

$\triangle$ Fig. 12b : pose de la prothèse en février 2014.

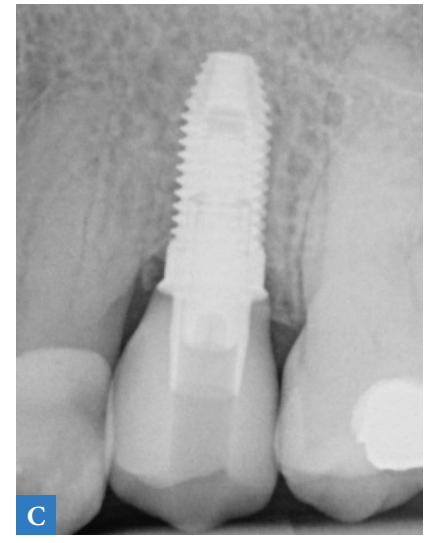

$\triangle$ Fig. 12c: contrôle radiographique en février 2017.

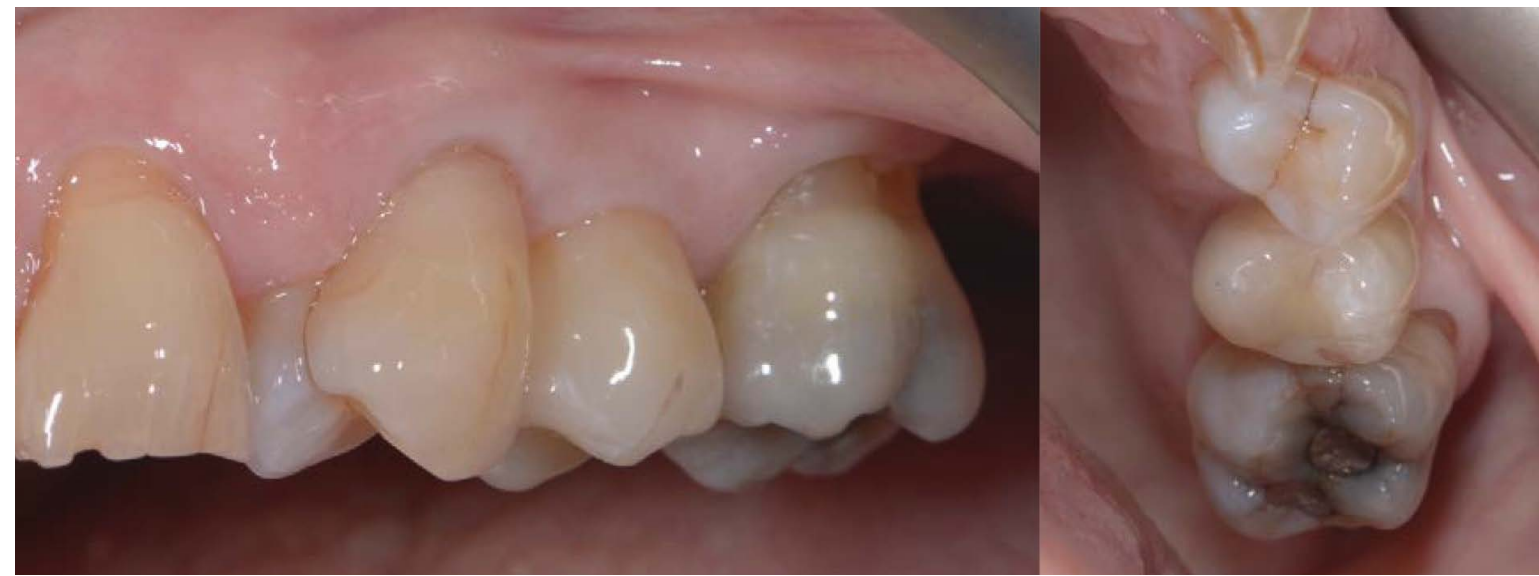

$\triangle$ Fig. 13 : vues cliniques vestibulaire et occlusale après 3 ans. Maquillage de la céramique réalisé par Mme Marie Pili, assistante dentaire. 
Cas clinique ${ }^{\circ} 3$ (Fig. 14 à 19, Fig. 20a, b, c, d, e, Fig. 21, Fig. 22)

Couronne pilier hybride en IPS e $\max ^{\circ}$ stratifiée en céramique feldspathique par la technique du cut-back (réduction homothétique vestibulaire après usinage). Stratification cosmétique réalisée par Mme Houda Beji, prothésiste dentaire.

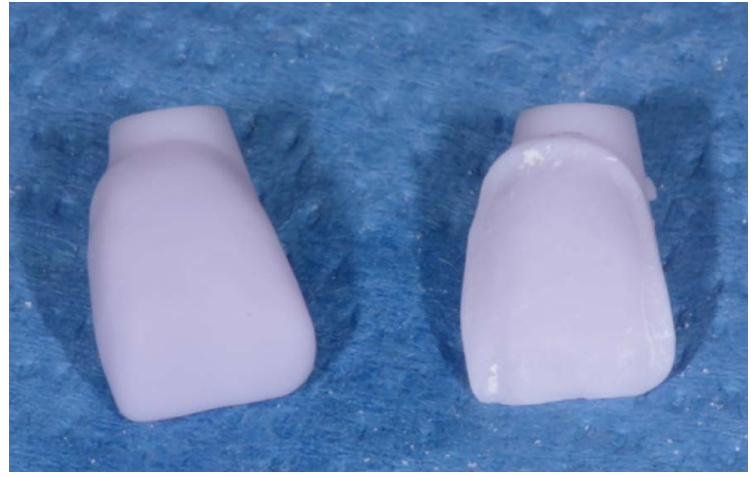

Fig. 14.

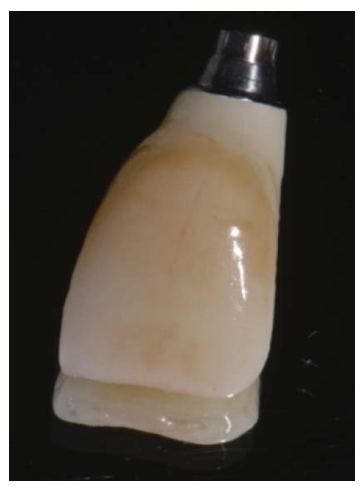

Fig. 17.

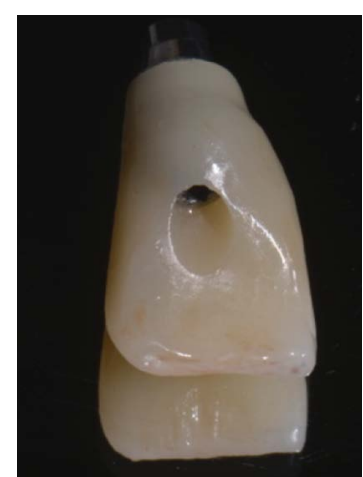

$\triangle$ Fig. 18.

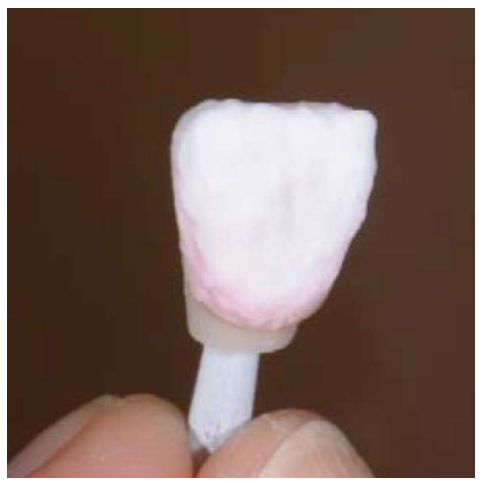

Fig. 15.

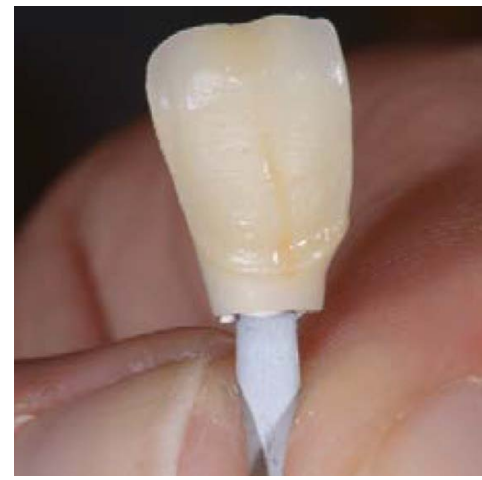

$\triangle$ Fig 16 .

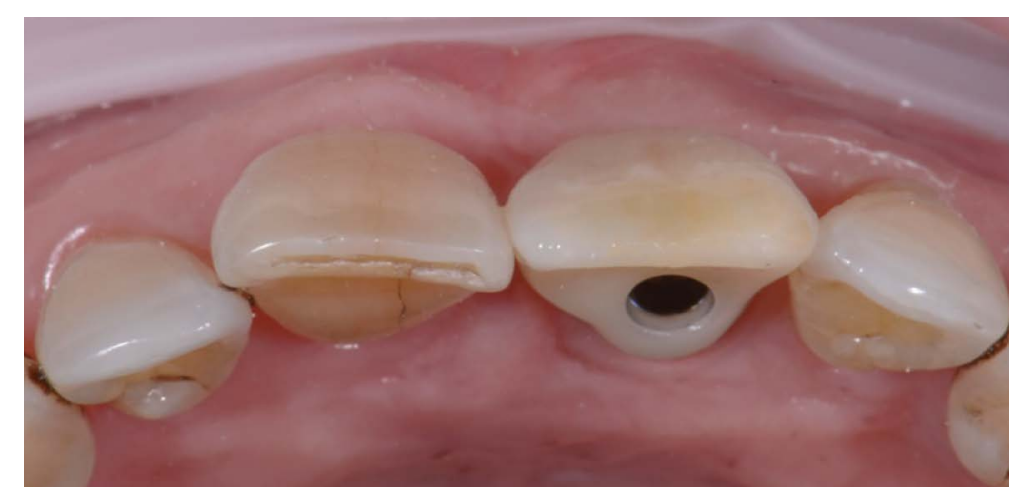

Fig. 19.

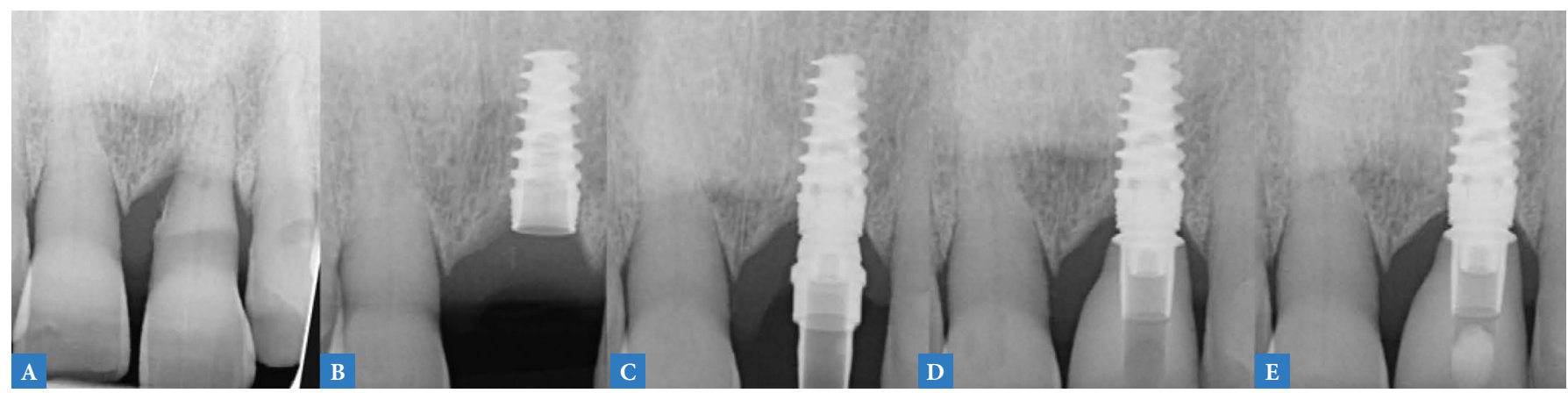

Fig. 20 : Suivi radiographique :

a : avant avulsion de 21 ;

b : pose de l'implant ;

c : prise d'empreinte à 3 mois ;

d: contrôle de la pose de la couronne pilier hybride ;

e : contrôle 18 mois après la pose de la prothèse. 


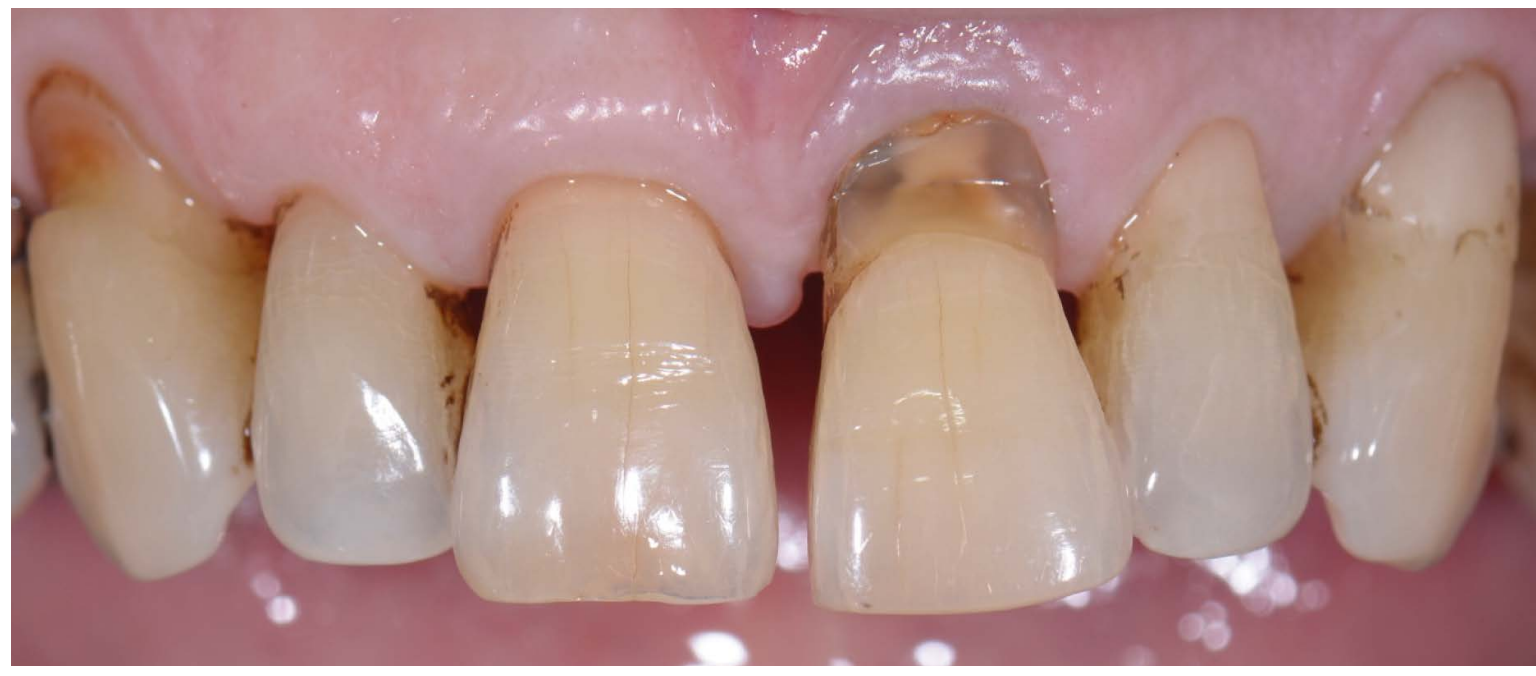

$\triangle$ Fig. 21 : Situation initiale avant avulsion de 21.

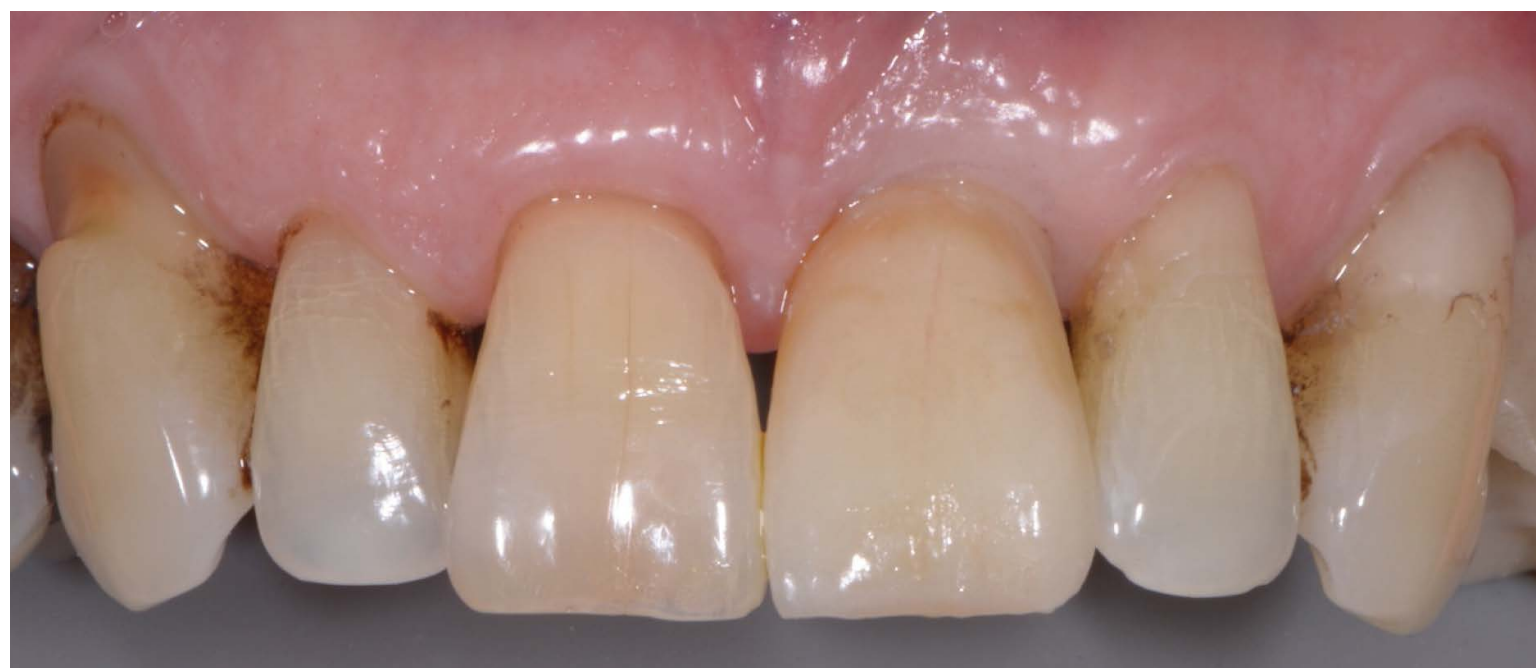

$\triangle$ Fig. 22 :Mise en place de la prothèse implanto-portée.

Cas clinique $n^{\circ} 4$ (Fig. 23, Fig. 24, Fig. 25, Fig. 26, Fig. 27, Fig. 28a, b, c)

Piliers hybrides fabriqués et placés le jour de la pose des implants à un niveau infra crestal. Lors de la cicatrisation, l'espace biologique s'est organisé au contact de l'assemblage prothétique sans altérer le niveau osseux crestal comme le montre ce cliché réalisé 1 an après la pose.

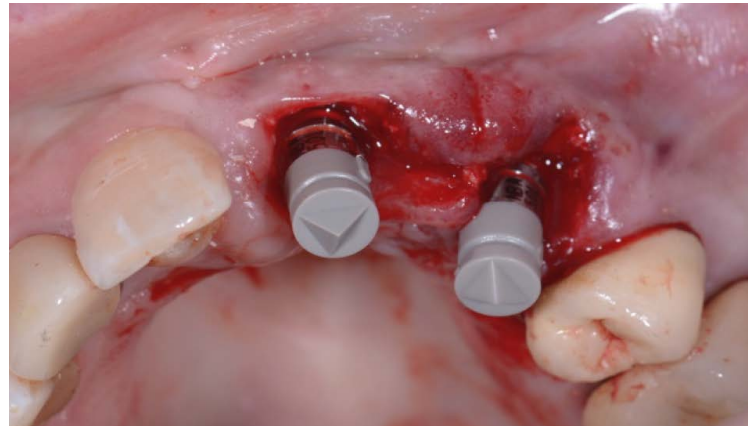

$\triangle$ Fig. 23 : empreinte optique extemporanée.

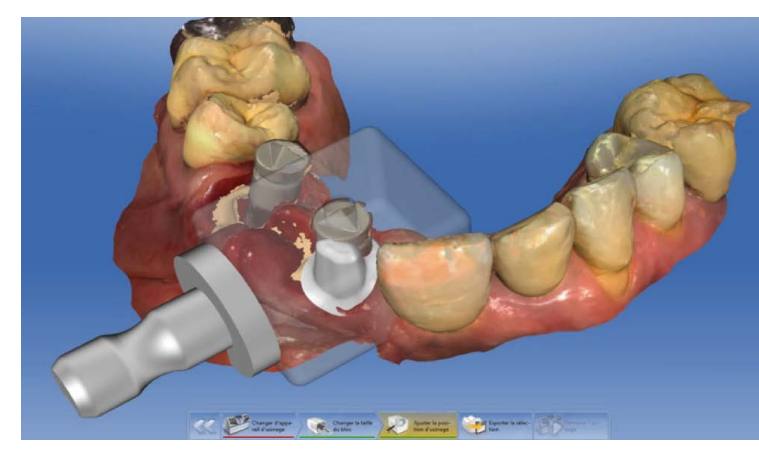

$\triangle$ Fig. 24 : conception des piliers et du bridge provisoire pour mise en place immédiate avec le logiciel Cerec Premium. 


\section{Numéro spécial CFAO et Zircone Drogou $\mathrm{H}$.}

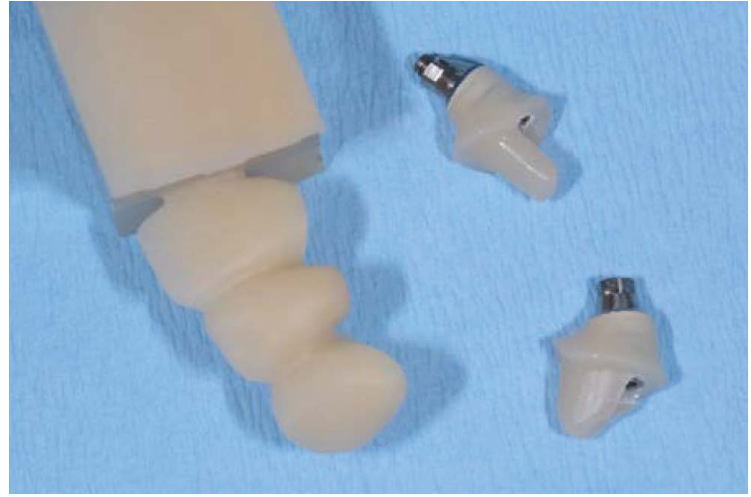

$\triangle$ Fig. 25 : piliers hybrides de 21 et 23 et bridge temporaire usiné en pmma à partir de l'empreinte optique extemporanée.

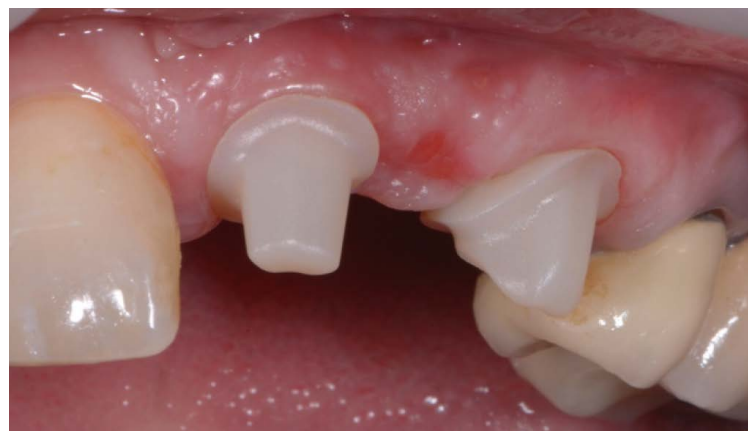

$\triangle$ Fig. 27 : cicatrisation à 3 mois après dépose du bridge temporaire. Les piliers seront retouchés en bouche pour ajuster les limites prothétiques à un niveau légèrement supra gingival.

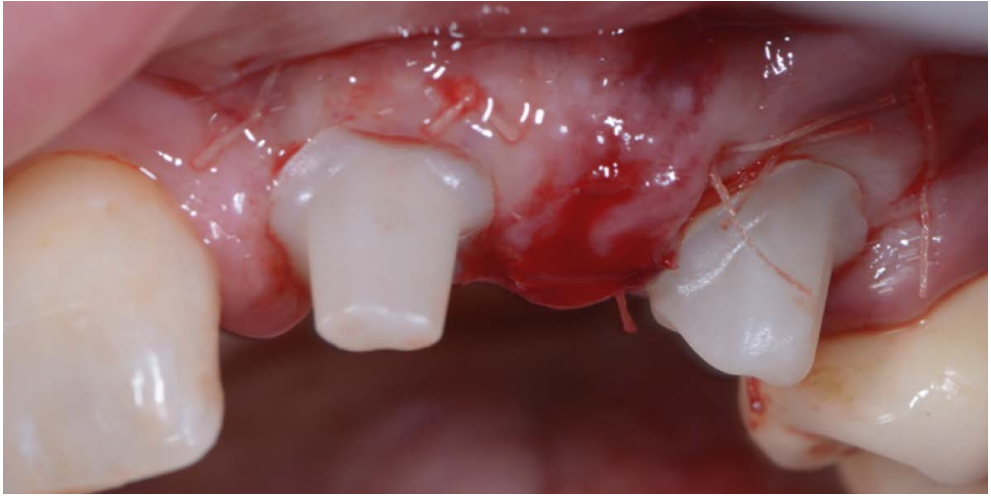

$\triangle$ Fig. 26 : vissage des piliers 6 heures après la pose des implants.
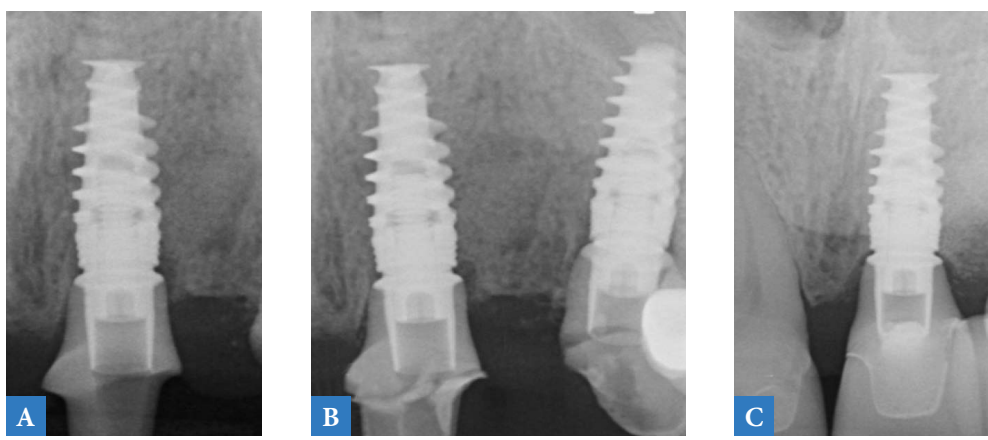

$\triangle$ Fig. 28 : Compilation des radiographies de contrôle:

a : vissage des piliers à $\mathrm{J} 0$;

b : contrôle à $J+7$;

c: contrôle à 1 an.

Cas clinique ${ }^{\circ} 5$ (Fig. 29, Fig. 30, Fig. 31)

Piliers hybrides en zircone réalisés à partir d'une empreinte optique. Conçus à l'aide du logiciel Inlab $15^{\circ}$, ils ont été usinés par le laboratoire Argoat Prothèses Dentaires.

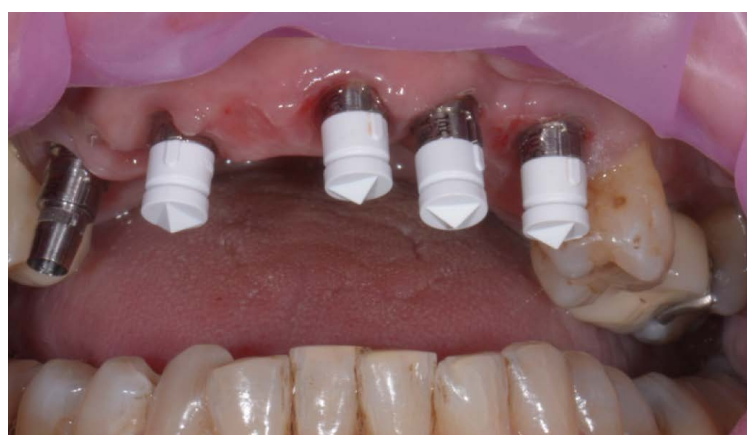

$\triangleleft$ Fig. 29: Transferts d'empreintes et scan bodies en place pour l'empreinte optique avec l'Omnicam ${ }^{\circ}$ (Dentsply-Sirona $\left.{ }^{\circ}\right)$.
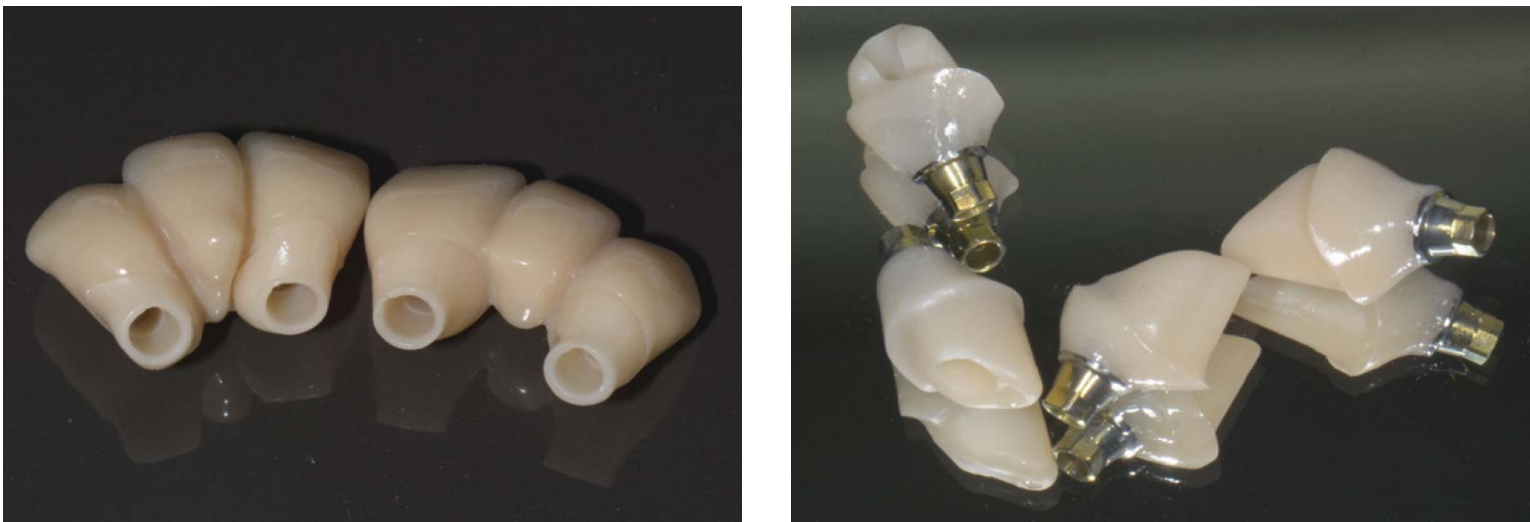

$\triangle$ Fig. 30 et 31 : Bridge provisoire en PMMA usiné et piliers implantaires en zircone avant et après assemblage avec les TiBases". 


\section{CONCLUSION}

Les piliers hybrides ou les couronnes piliers hybrides sur implants réalisés par CFAO directe recueillent la satisfaction des utilisateurs alors que les publications scientifiques les concernant sont rares. Les exemples cliniques présentés ici témoignent de 3 ans et demi de pratique d'un cabinet dentaire libéral. A l'exception de deux cas d'erreur de protocole, aucune fracture de céramique n'a été observée. La tolérance tissulaire est également cliniquement satisfaisante, à condition d'adopter des protocoles de fabrication et de pose très rigoureux. Certaines étapes de la fabrication peuvent être déléguées au sein du cabinet dentaire ou dans un laboratoire de prothèse, mais le contrôle de la qualité de fabrication incombe au chirurgien-dentiste qui est maintenant en mesure de gérer le processus du début à la fin.

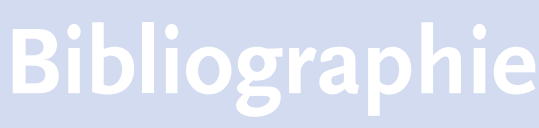

[1] Kurbad A, Kurbad S. CAD/CAM-based implant abutments. Int J Comput Dent. 2013;16(2):125-41.

[2] Food and Drug Administration Decision Date: 10/31/2013 Device Classification Name: Abutment, Implant, Dental, Endosseous 510 (K) Number K132209 Device Name: IPS E.MAX CAD ABUTMENT SOLUTIONS

[3] Bertolini Mde M, Kempen J, Lourenço EJ, Telles Dde M. The use of CAD/CAM technology to fabricate a custom ceramic implant abutment: a clinical report. J Prosthet Dent. 2014;111(5):362-6

[4] Elsayed A, Wille S, Al-Akhali M, Kern M. Comparison of fracture strength and failure mode of different ceramic implant abutments. J Prosthet Dent. 2016 Oct 18.

[5] Weyhrauch M, Igiel C, Scheller H, Weibrich G, Lehmann KM. Fracture Strength of Monolithic All-Ceramic Crowns on Titanium Implant Abutments. Int J Oral Maxillofac Implants. 2016;31(2):304-9.

[6] Brackett MG, Lockwood P, Messer R, Lewis JB, Bouillaguet $S$, Wataha JC. In vitro cytotoxic response to lithium disilicate dental ceramics. Dental Materials. 2008;24(4); 450-456

[7] Brunot-Gohin C, Duval J-L, Verbeke S, Belanger K, Pezron I, Kugel G, Laurent-Maquin D, Gangloff S, Egles C. Biocompatibility study of lithium disilicate and zirconium oxide ceramics for esthetic dental abutments. J Periodontal Implant Sci. 2016 Dec;46(6):362-371
[8] Brunot-Gohin C, Duval J-L, Azogui E-E, Jannetta R, Pezron I, Laurent-Maquin D, Gangloff S-C, Egles C. Soft tissue adhesion of polished versus glazed lithium disilicate ceramic for dental applications. Dental Materials. 2013;29(9);205-212.

[9] Morãguez OD, Belser UC. The use of polytétrafluoroéthylène tape for the management of screw access channels in implant-supported prostheses. J Prosthet Dent. 2010;103(3):189-191.

[10] Moussally C. 2014

http://www.edp-dentaire.fr/clinique/ parodontologie/1301-quand-1-implantologierencontre-la-CFAO

[11] Skramstad M. 2014

http://www.cerecdoctors.com/blog/view/id/738/ emax-cad-abutments-what-do-you-need 